\title{
A SIMPLE TECHNIQUE FOR PALM RECOGNITION USING MAJOR LINES
}

\author{
V. KUMAR ${ }^{1}$, A. DUA ${ }^{1}$, H. BANSAL ${ }^{1}$, H. AGGARWAL ${ }^{1}$, A. MADAN ${ }^{2}$, J.S. BHATIA ${ }^{2}$ \\ ${ }^{1}$ Department of Electronics and Communication Engineering, Thapar University, Patiala. \\ ${ }^{2}$ Department of Electronics and Communication Engineering, Jaypee University of Information Technology, Solan. \\ E-mail: vinay.kumar@thapar.edu
}

\begin{abstract}
Present manuscript deals with the biometric identification and security. Features exploited for the purpose are the principal lines of the palm of an individual. We developed an algorithm which extracts palm region from a human hand, calculates few palm specific parameters using only principal lines that differentiate one palm from another and then verifies it against a database which contain $s$ the palms of the registered users.
\end{abstract}

Keywords: filtering, edge detection, feature extraction

\section{INTRODUCTION}

Palm recognition has become increasingly prevalent in modern biometric identification and verification systems $[1,2,3]$. This approach uses inhere nt biological features on the palm of each individual. The basic idea behind authentication through palm recognition is that no two palms are alike [4]. As palms have both uniqueness and permanence, they can be used as a trusted form of identification.

Of all the biometrics studied, palm-print has an advantage over other biometrics such as voice and face recognition where uniqueness between people is doubtful, or fingerprint and iris pattern where high-resolution images are required [5, 6]. Palm-prints are unique between people and relatively low-resolution images will suffice. Moreover, palm is less prone to injuries and spoofing, because of fewer intrusions a s compared to other scans. Palm has less intrusions as compared to other scans especially when the detection is done using the principal lines present on the palm [5]. Although the present algorithm is not suitable for sophisticated applications, where iris recognition is preferred, but it is an economical (we use low resolution camera for capturing images) substitute for identification of persons in a classroom, small meeting, party, small companies etc.

Palm recognition technique can be used for security enhancement, authentication purposes and in some other management systems. It can be extended for sophisticated systems by including it in the multilayer recognition systems consisting of iris recognition, palm geometry, palm wrinkles, fingure ridges, etc.

The design methods proposed till now are based on vein and ridge patterns on figures and palm. These techniques produce anomalous results because of the change in vein and ridge patterns with time. In addition to that, they are time consuming, difficult and costly to design [7]. It is important to note that the sensor cost for capturing palm features is very less as compared to other biometric systems. In the present manuscript we use simple handheld mobile camera for capturing palm images.

We have developed an algorithm, which can be used to recognize palms for various purposes. The proposed design is based on inherent mathematical features present on the palm. The design methodology is based only on the principal lines of the hand. Since image acquisition does not requires any special high precision sensors the proposed method-ology becomes very cost effective. We have formed some definite patterns through the principal lines; which are unique for each palm. Figure 1 shows the principal lines of a palm.

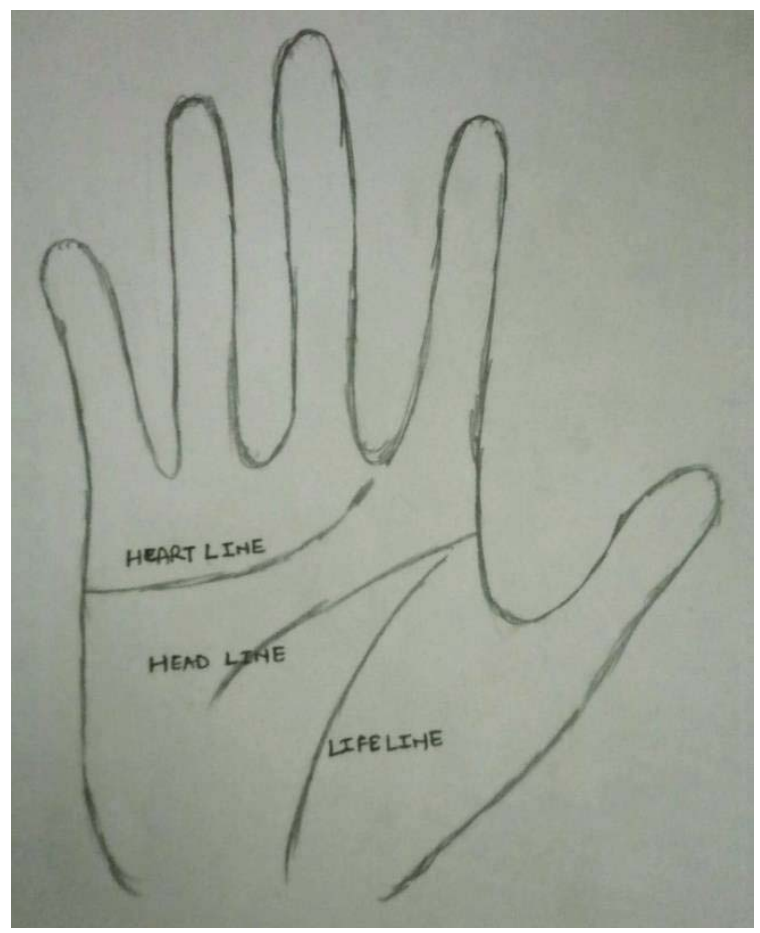

Figure 1. Principle lines on a palm.

Rest of the manuscript is divided into 3 sections. Section 2 of the manuscript details about the procedure while Section 3 and 4 presents results and the conclusion, respectively.

\section{PROPOSED METHOD}

The proposed method is outlined in the flowchart of Figure 2. 
The first step is to get a database of palm images. For uniformity, the images are captured under natural light, room light (with no special consideration for direction of light) with a handheld mobile camera of 13.0 megapixel. These images undergo following steps to create a database:

(1) Edge detection,

(2) Palm extraction,

(3) Pattern formation,

(4) Database formation, and

(5) Pattern matching for identification.

These modules are discussed in the successive subsections.

\subsection{Edge Detection.}

A sudden change in the intensity value of the pixel [8] is defined as edge of the image. The edge detection process is based on the concept of detecting and extracting the principal lines.

In order to get the result which is independent of orientation, we used the concept of gradients $[9,10]$. Gradients are used for calculating the strength of palm edges. Prewitt edge detector is used for gradient based edge detection. The algorithm for edge detection is discussed in Algorithm 1.

\section{Algorithm 1 Edge Detection Algorithm}

(1) Capture a grayscale image (or convert color to grayscale).

(2) Use directional operators $H_{x}$ and $H_{y}$ to produce gradient images $D_{x}$ and $D_{y}$, respectively.

(3) Calculate edge strength matrix.

(4) Calculate the level for threshold, th. It is given by th $=\square\left(I_{\max }-I_{\min }\right)+I_{\min }$; where $I_{\min }$ and $I_{\max }$ are minimum and maximum values in edge strength matrix, respectively.

(5) Store the maximum of edge strength or threshold level in a new matrix $i b w$.

(6) If $i b w(I, j)>t h$

- If the elements in $D_{x}$ and $D_{y}$ matrices have value greater than $i b w(I, j)$, then $(i, j)$ position of matrix item $p$ is assigned value imax; i.e., maximum edge strength.

- If the elements in $D_{x}$ and $D_{y}$ matrices have value less than $i b w(I, j)$, then $(i, j)$ position of matrix item $p$ is assigned value imin; i.e., minimum edge strength.

(7) If $i b w(I, j) \leq t h(i, j)$ position of matrix itemp is assigned value $I_{\min }$. Thus item $p$ gives us the extracted edges.

\footnotetext{
Algorithm 2 Palm extraction.

(1) Get Image $I$.

(2) Apply Laplacian operators to get the binary palm region.

(3) Define and initialize the variables am, bm, sqrside, sqrsidem equal to 0 .

(4) Variable sqrsidem stores the value of the size of the maximum square formed till now.

(5) The sqrside variable is changed dynamically in a pixel by pixel operation and the final result is stored in the sqrsidem variable.
}

(6) A square with side equal to sqrsidem is constructed with starting pixel co-ordinates as $(a m, b m)$.

(7) The co-ordinates $(a m, b m)$ along with sqrsidem are used to get the portion of palm from the hand image.

(8) The resulting image is the 'extracted palm'.

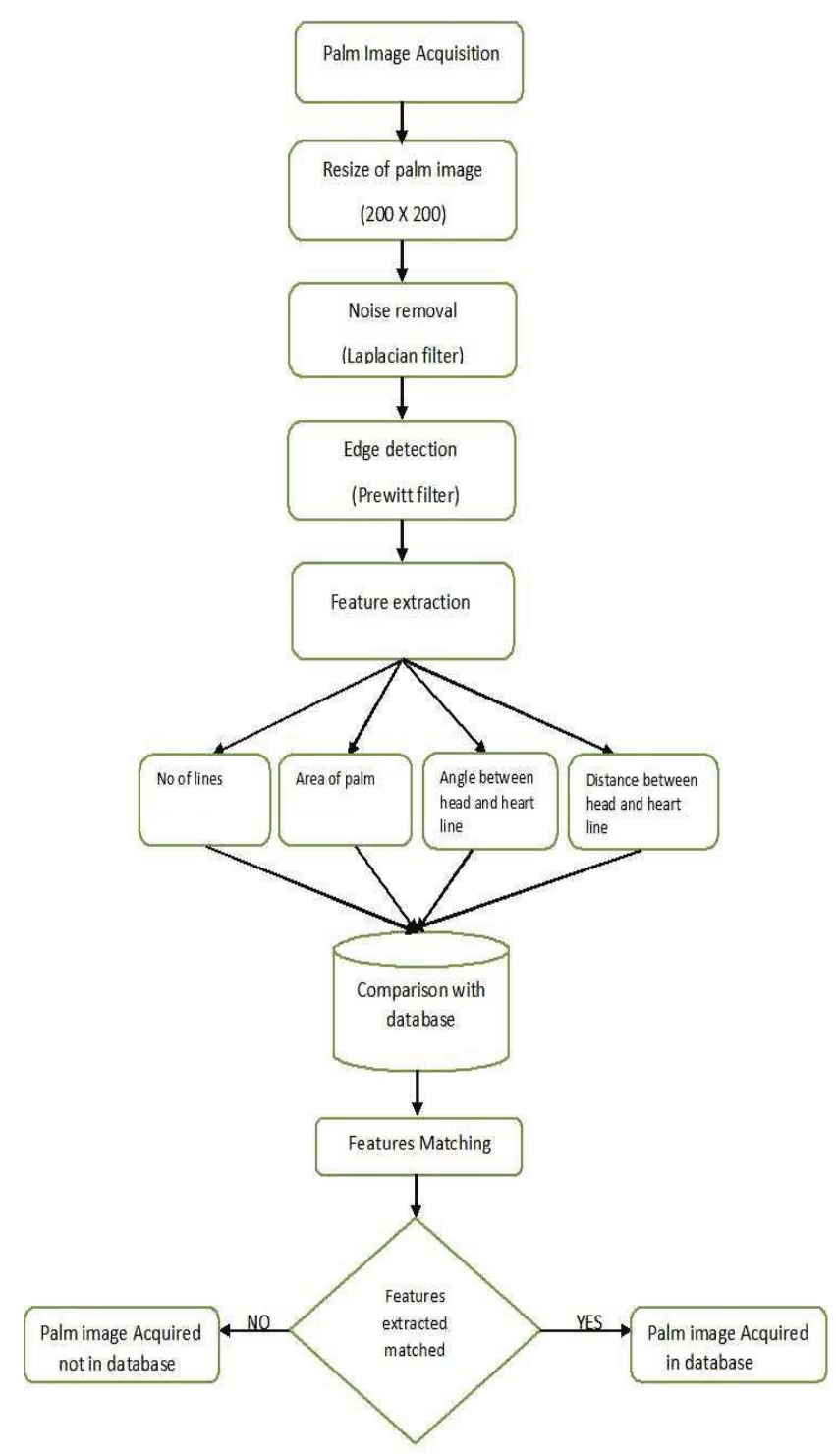

Figure 2. The algorithm

Results after edge detection are shown in Figure 3. In the present case application of thinning [11] removes any unwanted information from the binary image. 

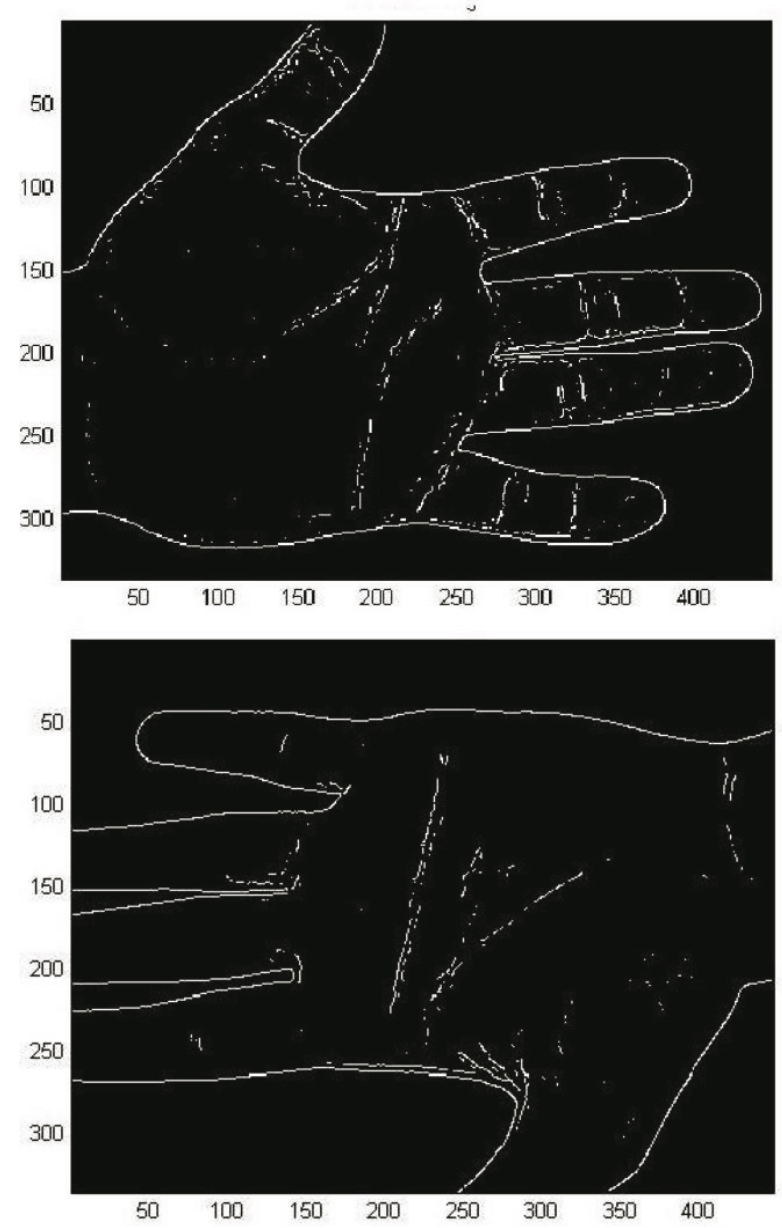

Figure 3. Edge detection results after threshold and thinning.

\subsection{Palm extraction.}

The actual palm area is called the Region Of Interest (ROI) as depicted in Figure 4. We use ROI to obtain characteristic parameters of the palm, and analyze them for identification.

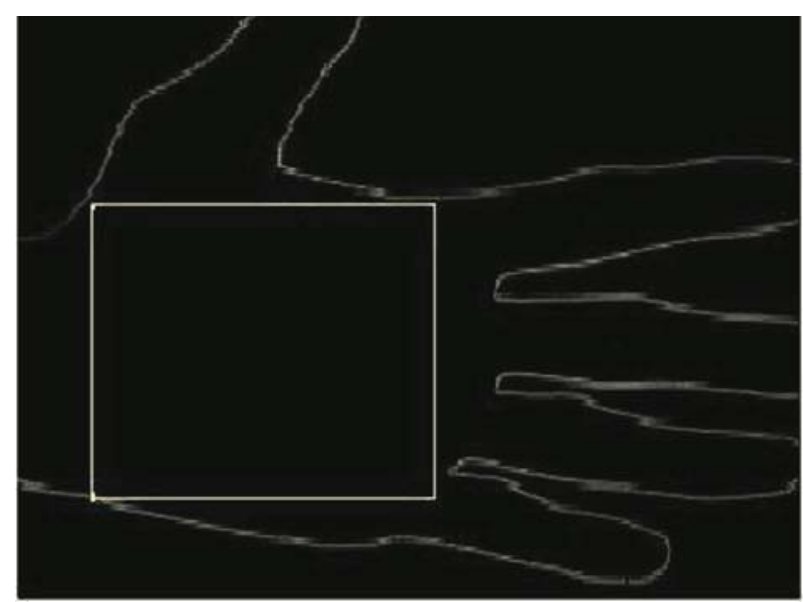

Figure 4. Region of interest of a palm.

First the outline of the hand is calculated with the help of Laplacian operators [8]. The stepwise algorithm for the extraction of the palm is discussed in Algorithm 2 (the pictorial representation for the algorithm is shown in Figure 5).

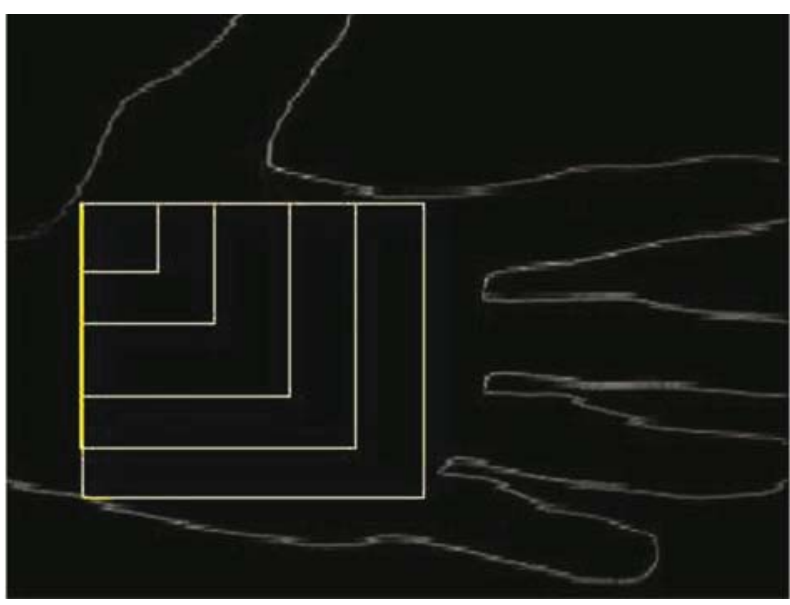

Figure 5. Iterative palm extraction using ROI

\subsection{Pattern Formation.}

One of the important factors for this kind of recognition system is pattern formation [12]. The criteria used to recognize the uniqueness of the palms is as follows:

(1) The number of lines extracted during edge extraction.

(2) The perpendicular distance between the head line and the heart line.

(3) The angle between lines formed by head line and heart line.

(4) The area of the palm extracted.

These features are discussed in detail in the following subsections.

(1) Perform edge detection and palm extraction.

(2) Draw a line between $(0,0)$ and (sqrsidem, sqrsidem) (Figure 6).

(3) Check for the points where this line intersects with black pixels.

(4) Store these points in an array.

(5) The number of entries in this array gives the number of lines on the palm.

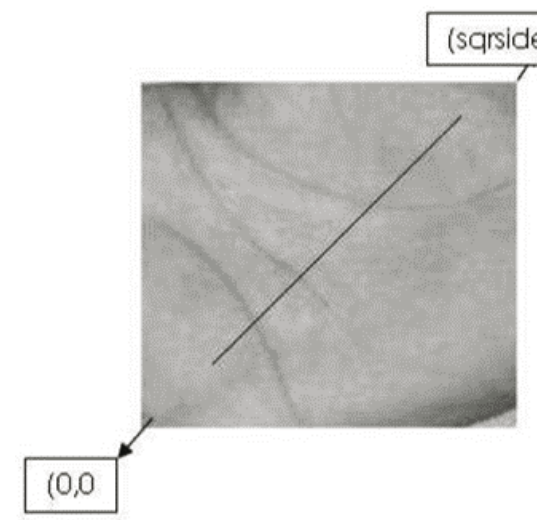

Figure 6. Number of Lines

2.3.1. Number of lines. The number of lines extracted after the edge detection process are calculated. This value is 
generally between 5 and 15 . It has been observed that palm samples may have different number of prominent lines, which is used as a distinguishing feature for different palms.

The algorithm to perform this operation is discussed in Algorithm 3.

2.3.2. Distance between the head and the heart line. Next we calculate the distance be-tween head line and heart line. This distance is calculated in pixels, which gives us a fairly sufficient amount of sample space. The numeric value of distance obtained generally stood between 10 to 70 pixels.

Algorithm 4 Distance between heart and head line.

(1) On the extracted ROI (Figure 7) traverse to half the length in vertical direction.

(2) Draw a line perpendicular to vertical axis from that point.

(3) This line intersects the heart line and head line at two points, say $A$ and $B$ respectively.

(4) We calculate the distance $d$ between points $A\left(x_{1}\right.$, $\left.y_{1}\right)$ and $B\left(x_{2}, y_{2}\right)$ using distance formula.

$$
d=\sqrt{\left(x_{1}-x_{2}\right)^{2}+\left(y_{1}-y_{2}\right)^{2}}
$$

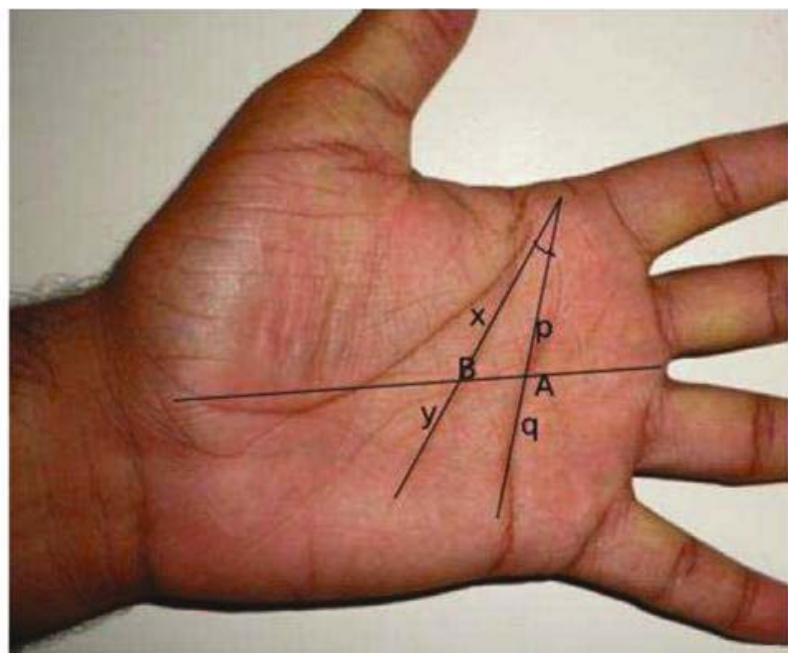

Figure 7. Angle Calculation.

Algorithm to calculate the distance between the head and the heart line is discussed in Algorithm 4.

This distance is measured in pixels.

2.3.3. Angle between the head line and the heart line [13]. If the head line and the heart line are extrapolated, they intersect at some point and the angle at the point of intersection can be used as a distinguishing feature. This value generally varies between 0 to 45 degrees.

From Figure 7 we can find that:

(1) The principal axis intersects the heart line and the head line, say at points A and B respectively.

(2) Points $p$ and $q$ lie on the heart line whereas $x$ and $\mathrm{y}$ lie on the head line. Points $\mathrm{p}$ and $\mathrm{q}$ are equidistant from point $\mathrm{A}$ and $\mathrm{x} \& \mathrm{y}$ are equidistant from point $\mathrm{B}$.

(3) We form a line using points $p$ and $q$ and another line using points $\mathrm{x}$ and $\mathrm{y}$.

(4) Then, finally we find the angle between 2 lines obtained. This angle shall work as the distinguishing factor between palms. We calculated the values of $\mathrm{p}, \mathrm{q}, \mathrm{x}$ and $\mathrm{y}$ as follows.

$$
\begin{gathered}
p=A+10 \text { (pixels) } \\
q=A-10 \text { (pixels) } \\
x=B+10 \text { (pixels) } \\
y=B-10 \text { (pixels) }
\end{gathered}
$$

The algorithm for calculating the angle is discussed in Algorithm 5.

The $\theta$ value is in radians.

Algorithm 5 Calculation of angle between head and heart line.

(1) First slopes $s_{1}$ and $s_{2}$ of the lines $p q$ and $x y$, respectively, are calculated.

(2) Then the slopes are used to calculate angle of intersection with following formula:

$$
\theta=\tan ^{-1}\left(\left(s_{1}-s_{2}\right) /\left(1+s_{1} \times s_{2}\right)\right)
$$

2.3.4. Area of the palm extracted. The area of palm region is calculated by taking square of variable sqrsidem and unit of area is pixel $\times$ pixel.

\subsection{Pattern Matching}

Pattern matching compares palm of the user, trying to access the system, with those stored in the database and decide whether the user should be authenticated or not.

A database is created with following entries as columns of a matrix:

(1) Number of Lines

(2) Distance between the head and the heart line (in Pixels)

(3) Angle (in degrees)

(4) Area (in thousand pixels ${ }^{2}$ ) 


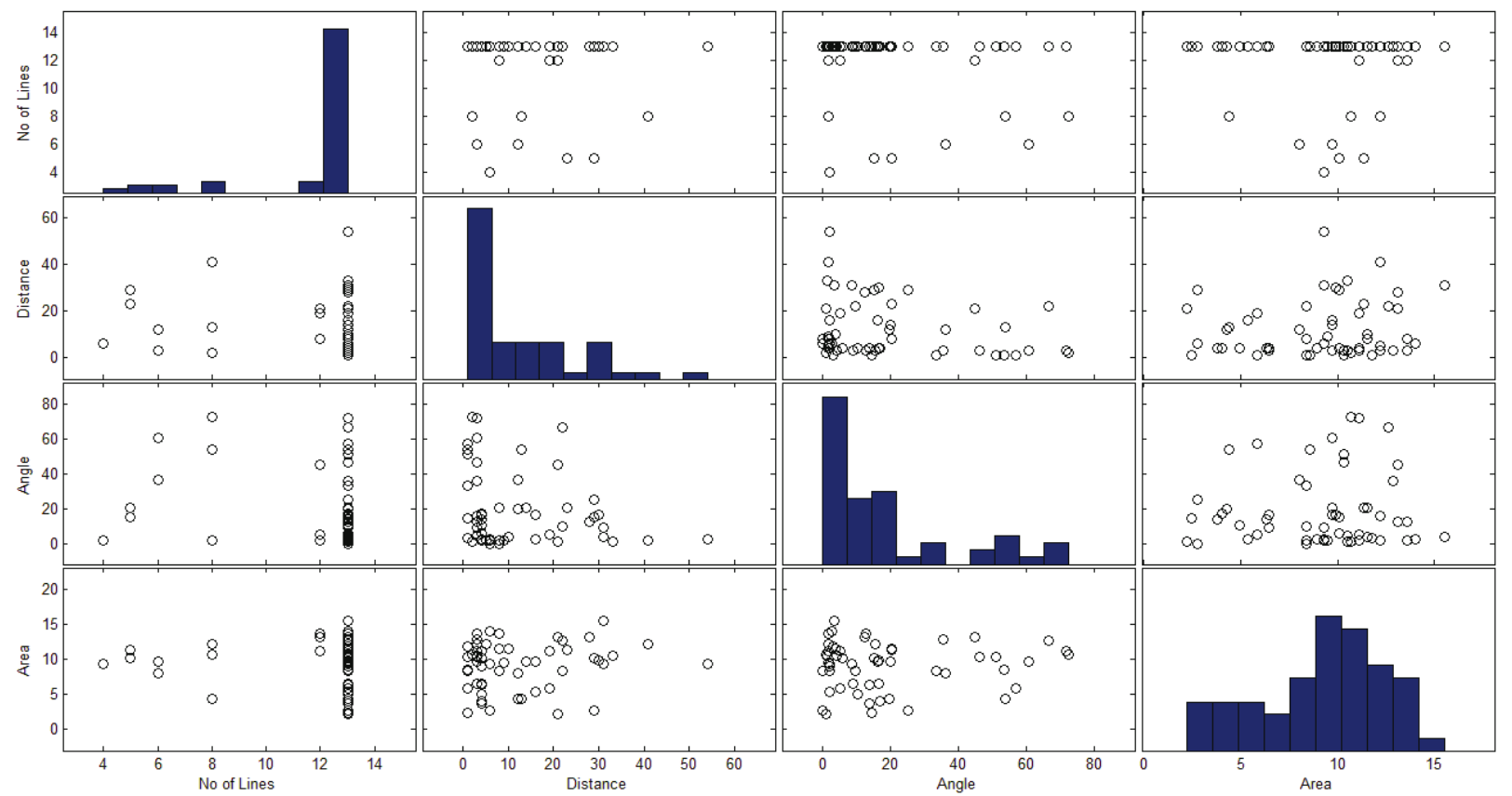

Figure 8. Palm Comparison.

\section{RESULTS}

We applied our algorithm for a database consisting of 100 palms. Table 1 consists of a sample of calculations for 20 palms. Figure 8 indicates that various palms, results for 55 palms, have different characteristics and based on four previously discussed parameters one can easily identify the person. $\mathrm{X}$ and $\mathrm{Y}$-axis indicates the features, and their histogram on diagonal; which their mutual dependance is indicated on non diagonal areas. For example, it becomes evident that number of lines with angle or distance segregates only few palms. Palms not recognized with said relationship are further segregates with the help of mutual dependence of rest of features, refer Figure 9 for mutual dependence of parameters.

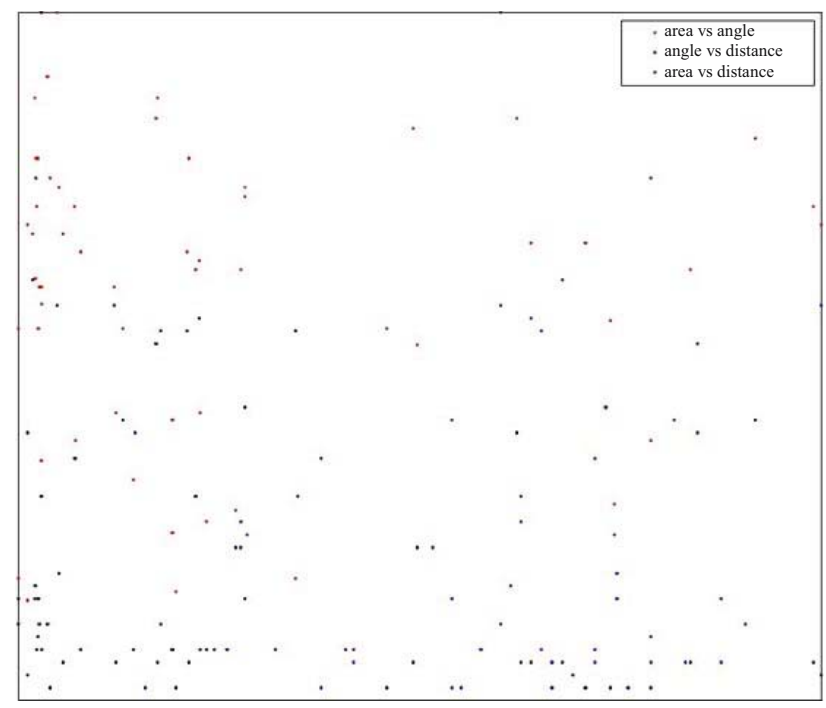

Figure 9. Palm Comparison with Features
Table 1. Sample Palm Database

\begin{tabular}{|c|c|c|c|c|}
\hline $\begin{array}{c}\text { Sr } \\
\text { No. }\end{array}$ & $\begin{array}{c}\text { No } \\
\text { Of } \\
\text { Lines } \\
\end{array}$ & $\begin{array}{c}\text { Distance } \\
\text { between } \\
\text { Lines } \\
\end{array}$ & Angle & Area \\
\hline 1 & 4 & 6 & 1.909152 & 9.31225 \\
\hline 2 & 5 & 29 & 15.25512 & 10.10025 \\
\hline 3 & 5 & 23 & 20.47784 & 11.34225 \\
\hline 4 & 6 & 3 & 60.6655 & 9.70225 \\
\hline 5 & 6 & 12 & 36.02737 & 8.01025 \\
\hline 6 & 8 & 41 & 1.618095 & 12.21025 \\
\hline 7 & 8 & 13 & 53.82386 & 4.42225 \\
\hline 8 & 8 & 2 & 72.50424 & 10.71225 \\
\hline 9 & 12 & 19 & 5.102165 & 11.13025 \\
\hline 10 & 12 & 8 & 1.507436 & 13.57225 \\
\hline 11 & 12 & 21 & 45 & 13.11025 \\
\hline 12 & 13 & 3 & 8.826371 & 6.48025 \\
\hline 13 & 13 & 22 & 66.5385 & 12.65625 \\
\hline 14 & 13 & 1 & 33.27033 & 8.37225 \\
\hline 15 & 13 & 1 & 14.26451 & 2.45025 \\
\hline 16 & 13 & 19 & 5.204886 & 5.85225 \\
\hline 17 & 13 & 54 & 2.102425 & 9.31225 \\
\hline 18 & 13 & 16 & 2.102425 & 5.40225 \\
\hline 19 & 13 & 4 & 16.41565 & 6.48025 \\
\hline 20 & 13 & 12 & 19.61462 & 4.29025 \\
\hline
\end{tabular}




\section{CONCLUSION AND FUTURE WORK}

The possession of access control is very important when confidential, or sensitive information and equipment is to be secured. The present algorithm it is not exactly biometric in classical sense. While biometric is considers usage of some biological property of the per-son (and that is what makes them costly), whereas present technique can be applied using a low cost camera and is not dependent on any specific sensor re quirement. The current manuscript is meant to be used primarily for identity access management to authenticate users and grant or deny access rights to data or services provided by an organization. It can be extened for usage in authorization system for front doors, banks, airports, hospital wards, storage areas and schools.

\section{REFERENCES}

[1] Z. Sun, T. Tan, Y. Wang, and S. Z. Li, "Ordinal palmprint representation for personal identification," in Proc. IEEE Computer Vision and Pattern Recognition (CVPR, 2005, pp. 279-284.

[2] A. Jain, A. Ross, and S. Prabhakar, "An introduction to biometric recognition," Circuits and Systems for Video Technology, IEEE Transactions on, vol. 14, no. 1 , pp. $4-20$, jan. 2004.

[3] E. Yörük, H. Dutagaci, and B. Sankur, "Hand biometrics," Image Vision Comput., vol. 24, no. 5, pp. 483-497, 2006.

[4] A. Kong, D. Zhang, and M. Kamel, "A survey of palmprint recognition," pattern recognition, vol. 42, no. 7, pp. 1408-1418, 2009.

[5] A. Kumar, D. C. M. Wong, H. C. Shen, and A. K. Jain, "Personal verification using palmprint and hand geometry biometric," in Audio-and Video-Based Biometric Person Authentication. Springer Berlin Heidelberg, 2003, pp. 668-678.

[6] V. I. Pavlovic, R. Sharma, and T. S. Huang, "Visual interpr etation of hand gestures for human-computer interaction: A review," Pattern Analysis and Machine Intelligence, IEEE Transactions on, vol. 19, no. 7, pp. 677-695, 1997.

[7] N. Bhanushali, P. Nikam, P. Tripathi et al., "High security using palm vein recognition technology," Inter-national Journal of Computer Applications, vol. 119, no. 1, 2015.

[8] R. C. Gonzalez and R. E. Woods., Digital Image Processing. Prentice Hall, 2008.

[9] M. Basu, "Gaussian-based edge-detection methods-a survey," IEEE Transactions on Systems, Man, and Cybernetics, Part C (Applications and Reviews), vol. 32, no. 3, pp. 252-260, 2002.

[10] N. Senthilkumaran and R. Rajesh, "Image segmentation-a survey of soft computing approaches," in Advances in Recent Technologies in Communication and Computing, 2009. ARTCom'09. International Conference on. IEEE, 2009, pp. 844846.
[11] A. Rosenfeld and L. S. Davis, "A note on thinning," Systems, Man and Cybernetics, IEEE Transactions on, no. 3, pp. 226-228, 1976.

[12] R. L. Zunkel and C. Campbell, "4 hand geometry based verification," Personal Identification in Networked Society, p. 87, 1999.

[13] A. Amin and S. Fischer, "A document skew detection method using the hough transform," vol. 3, no. 3, 2000, pp. 243-253. 